10. Anderson FA Jr, Goldhaber SZ, Tapson VF, et al; ENDORSE Investigators. Improving practices in US hospitals to prevent venous thromboembolism: lessons from ENDORSE. Am J Med. 2010;123(12):1099-1106.

11. Liu DSH, Lee MMW, Spelman T, et al. Medication chart intervention improves inpatient thromboembolism prophylaxis. Chest. 2012;141(3):632-641.

\section{Obstructive Sleep Apnea}

\section{The Elephant in the Cardiovascular Room}

D espite considerable advances in the relatively young field of sleep research, the importance of obstructive sleep apnea (OSA) remains underappreciated. A vast body of evidence based on animal, ${ }^{1}$ human cross-sectional and longitudinal research, ${ }^{2}$ and interventional studies ${ }^{3}$ suggests that OSA is associated with significant cardiovascular risk factors. In addition, new therapeutic targets are needed in the cardiovascular arena given that improvements in outcomes have plateaued in many studies. Unfortunately, however, OSA remains the "elephant in the room" and often is ignored even in high-risk patients. A telling example comes from the diabetes field, with recent data indicating that $86 \%$ of obese patients with type 2 diabetes also experience clinically significant OSA, ${ }^{4}$ with $<5 \%$ of these patients receiving OSA treatment 1 year after both the patient and his or her primary physician have received the diagnosis. ${ }^{5}$

The reasons that OSA is often overlooked as a potentially reversible cardiovascular risk factor are complex but probably lie in the small but growing number of mechanistic studies in this area. ${ }^{6,7}$ The profile of OSA also would be lifted by the execution of large-scale, multicenter, randomized controlled trials of continuous positive airway pressure (CPAP) with hard cardiovascular outcomes, which is a current research focus. The design of such trials presents an ethical challenge: Reductions in daytime sleepiness and neurocognitive impairment are likely to result from administering CPAP to symptomatic patients with OSA, which promotes a reluctance to randomize hypersomnolent patients (at risk for car accidents) to a longduration arm without active treatment. Restricting entry criteria to nonsleepy patients presents issues around CPAP adherence because such patients may not perceive symptomatic benefit, and there is some evidence that only very small reductions in BP result from CPAP in nonsleepy patients. ${ }^{8}$ Thus, a negative result in a large-scale randomized controlled trial of CPAP in asymptomatic patients with OSA may simply mean that those who would demonstrate the greatest improvement were systematically excluded during recruitment. Thus, surrogate outcome mea- sures that accurately predict fatal and nonfatal cardiovascular events are critical, allowing for shorter trial durations and, therefore, a reduced ethical dilemma.

In this issue of CHEST (see page 674), Colish et $\mathrm{al}^{9}$ present new evidence that CPAP treatment is associated with a reduction in right atrial and ventricular size as well as a reduction in left ventricular mass as demonstrated by both transthoracic echocardiography and cardiac MRI (CMR), with no concurrent changes evident in a range of cardiac biomarkers that were within normal ranges at baseline. Strengths of the study include the multiple follow-up visits $(3,6$, and 12 months after the initiation of CPAP) and the targeting of patients with a high Epworth Sleepiness Scale score at baseline. Such studies are crucial in defining the time period needed to see important cardiovascular morphologic and physiologic changes resulting from CPAP therapy in OSA. These data are critical to the design of future randomized protocols. Further, this study likely will have a substantial impact on clinicians and researchers outside the sleep field who are perhaps more likely to appreciate CMR outcome measures over polysomnography-derived outcomes, such as the apnea-hypopnea index. Left ventricular mass has been shown to predict future cardiac events ${ }^{10}$ and decreased survival in patients with heart failure. ${ }^{11}$ By demonstrating left ventricular remodeling with CPAP in an OSA sample, Colish et $\mathrm{al}^{9}$ have helped to fill this research gap.

Despite the obvious strengths of the study, it has some limitations. The lack of a control group does not allow the beneficial cardiac remodeling to be attributed to CPAP; the natural history of CMR measurements among the OSA population is unknown, and therefore, it is possible that some degree of changes may have occurred without the addition of CPAP. As in all uncontrolled studies, diet, exercise, changes in medications, and medication adherence could have been affected by close monitoring and, thus, could have affected the outcomes. CPAP adherence in this study was high, and the fact that patients who are willing and able to enroll in a research study and are adherent to CPAP also may be more likely to embrace positive lifestyle changes should not be overlooked. ${ }^{12}$ However, this "healthy user" effect also can complicate randomized controlled trials if imbalances occur in the two arms after randomization.

As with most novel research, the study by Colish et al ${ }^{9}$ has generated several questions for clinicians and scientists in the field. For the clinician, is CMR a useful clinical tool to monitor cardiovascular improvements, and will this approach help to improve CPAP adherence? Can pretreatment CMR act as a reliable marker for identifying patients at high cardiovascular risk, even possibly those with mild OSA, such 
that they can be rapidly provided with CPAP and comprehensive pharmacotherapy alongside intensive support to optimize adherence? Does the high cost of CMR outweigh these benefits in an era of health-care reform? For the scientist, what are the early pathologic and physiologic changes in the heart due to OSA, and what is the chronologic sequence and reversibility of these changes? Are there other surrogate cardiac imaging markers that could be used to detect adverse ventricular remodeling, even when chamber volumes, mass, and function appear normal? What duration of CPAP and level of adherence are required to see important changes in CMR? Can other OSA treatments, such as oral appliances, surgery, or alternative pressure modes, cause improvements in cardiac morphology and physiology similar to those seen with CPAP? Finally, if future studies are able to attribute irrefutably improvements seen with CMR to CPAP, are these changes predictive of hard cardiovascular end points such as myocardial infarction and stroke in OSA?

Clearly, much work remains in answering these questions and elucidating the mechanistic link between OSA and cardiovascular disease, with studies such as that by Colish et $\mathrm{al}^{9}$ paving the way for this future research. We applaud the authors for moving us one step closer to the widespread appreciation of OSA.

\section{Jessie P. Bakker, PhD \\ Bhavneesh Sharma, MD Atul Malhotra, MD, FCCP \\ Boston, MA}

\begin{abstract}
Affiliations: From the Division of Sleep Medicine, Harvard Medical School, Brigham \& Women’s Hospital.

Financial/nonfinancial disclosures: The authors have reported to CHEST the following conflicts of interest: Dr Malhotra has consulting and research income from the National Institutes of Health (NIH); American Heart Association (AHA); Philips Respironics Inc; Apnex Medical Inc; Apnicure, Inc; Sleep Group Solutions; Sleep HealthCenters; Ethicon, Inc; Medtronic, Inc; Pfizer Inc; Merck \& Co, Inc; Sepracor; Cephalon, Inc; and Galleon Pharmaceuticals. Dr Malhotra is the principal investigator for NIH R01 HL085188, NIH R01 HL090897, NIH K24 HL 093218, NIH P01 HL 095491, NIH R01 AG035117, and AHA 0840159N. Drs Bakker and Sharma have reported that no potential conflicts of interest exist with any companies/organizations whose products or services may be discussed in this article.

Correspondence to: Jessie P. Bakker, PhD, Division of Sleep Medicine, Harvard Medical School, Brigham \& Women's Hospital, 221 Longwood Ave, 036 BLI, Boston, MA 02115; e-mail: jpbakker@ partners.org

(C) 2012 American College of Chest Physicians. Reproduction of this article is prohibited without written permission from the American College of Chest Physicians (http://www.chestpubs.org/ site/misc/reprints.xhtml)
\end{abstract}

DOI: 10.1378/chest.11-2178

\section{REFERENCES}

1. Brooks D, Horner RL, Kozar LF, Render-Teixeira CL, Phillipson EA. Obstructive sleep apnea as a cause of systemic hypertension. Evidence from a canine model. J Clin Invest. 1997;99(1):106-109.
2. Young T, Finn L, Peppard PE, et al. Sleep disordered breathing and mortality: eighteen-year follow-up of the Wisconsin sleep cohort. Sleep. 2008;31(8):1071-1078.

3. Marin JM, Carrizo SJ, Vicente E, Agusti AG. Long-term cardiovascular outcomes in men with obstructive sleep apnoeahypopnoea with or without treatment with continuous positive airway pressure: an observational study. Lancet. 2005; 365(9464):1046-1053.

4. Foster GD, Sanders MH, Millman R, et al; Sleep AHEAD Research Group. Obstructive sleep apnea among obese patients with type 2 diabetes. Diabetes Care. 2009;32(6): 1017-1019.

5. Foster GD, Borradaile KE, Sanders MH, et al; Sleep AHEAD Research Group of Look AHEAD Research Group. A randomized study on the effect of weight loss on obstructive sleep apnea among obese patients with type 2 diabetes: the Sleep AHEAD study. Arch Intern Med. 2009;169(17): 1619-1626.

6. Büchner NJ, Quack I, Woznowski M, Stähle C, Wenzel U, Rum LC. Microvascular endothelial dysfunction in obstructive sleep apnea is caused by oxidative stress and improved by continuous positive airway pressure therapy. Respiration. 2011;82(5):409-417.

7. Rahangdale S, Yeh SY, Novack V, et al. The influence of intermittent hypoxemia on platelet activation in obese patients with obstructive sleep apnea. J Clin Sleep Med. 2011;7(2): 172-178.

8. Barbé F, Durán-Cantolla J, Capote F, et al; Spanish Sleep and Breathing Group. Long-term effect of continuous positive airway pressure in hypertensive patients with sleep apnea. Am J Respir Crit Care Med. 2010;181(7):718-726.

9. Colish J, Walker JR, Elmayergi N, et al. Obstructive sleep apnea: effects of continuous positive airway pressure on cardiac remodeling as assessed by cardiac biomarkers, echocardiography, and cardiac MRI. Chest. 2012;141(3): 674-681.

10. Bluemke DA, Kronmal RA, Lima JA, et al. The relationship of left ventricular mass and geometry to incident cardiovascular events: the MESA (Multi-Ethnic Study of Atherosclerosis) study. J Am Coll Cardiol. 2008;52(25):2148-2155.

11. Dini FL, Capozza P, Donati F, et al. Patterns of left ventricular remodeling in chronic heart failure: prevalence and prognostic implications. Am Heart J. 2011;161(6):1088-1095.

12. Platt AB, Kuna ST, Field SH, et al. Adherence to sleep apnea therapy and use of lipid-lowering drugs: a study of the healthy-user effect. Chest. 2010;137(1):102-108.

\section{The Lung Cancer Stage Page}

\section{There When You Need It-StagingLungCancer.org}

Tt seems that life gets more and more complicated; 1 this is true as well of the International Union Against Cancer (UICC) and American Joint Committee on Cancer (AJCC) seventh edition of the lung cancer staging system. The increased granularity brought about by the huge increase in the database and the underlying analysis has made the system less intuitive and difficult, if not impossible, to remember. ${ }^{1,2}$ Both for those who deal with lung cancer occasionally and for focused subspecialists, this increased level of 